\title{
Guanosine Mechanisms of Action: Toward Molecular Targets
}

\author{
Caio M. Massari ${ }^{1}$, Mariachiara Zuccarini ${ }^{2}$, Patrizia Di lorio ${ }^{2}$ and Carla I. Tasca ${ }^{1 *}$ \\ ${ }^{1}$ Laboratório De Neuroquímica-4, Departamento De Bioquímica, Centro De Ciências Biológicas, Universidade Federal De Santa \\ Catarina, Florianópolis, Brazil, ${ }^{2}$ Department of Biomedical Sciences, University G. D'Annunzio of Chieti-Pescara, Chieti, Italy
}

Keywords: guanosine, adenosine receptors, receptors oligomerization, A1R/A2AR heteromers, neuroprotection

\section{INTRODUCTION}

Over the years, increasing data concerning the extracellular effect of guanine-based purines have been published. This class of molecules that embraces the well-known nucleotides GTP, GDP and GMP, the nucleoside guanosine (GUO) and the nucleobase guanine are necessary for the maintenance of important intracellular processes, such as nucleic acid structure, energetic metabolism, and signal transduction via G-proteins (Hepler and Gilman 1992). Besides that, guanine-based purines extracellular effects are also noteworthy, mainly through its nucleoside GUO.

In the central nervous system (CNS), GUO acts as a neuromodulator mediating several cellular processes, including cell growth, differentiation and survival (Lanznaster, et al., 2016; Schmidt, Lara, and Souza 2007). Also, GUO exerts protective effects in several models of neurotoxicity or neurological disorders (both in vitro and in vivo). GUO presents anxiolytic (Bettio et al., 2014),

\section{OPEN ACCESS}

Edited by:

Kjell Fuxe,

Karolinska Institutet (KI), Sweden

Reviewed by:

Cesar Quiroz,

National Institute on Drug Abuse

(NIDA), United States

${ }^{*}$ Correspondence:

Carla I. Tasca

carla.tasca@ufsc.br

Specialty section:

This article was submitted to Experimental Pharmacology and Drug Discovery,

a section of the journal

Frontiers in Pharmacolog

Received: 13 January 2021 Accepted: 26 February 2021

Published: 31 March 2021

Citation:

Massari CM, Zuccarini M, Di lorio P and Tasca Cl (2021) Guanosine Mechanisms of Action: Toward Molecular Targets.

Front. Pharmacol. 12:653146. doi: 10.3389/fphar.2021.653146 antidepressant-like (Bettio et al., 2012), antinociceptive (Schmidt et al., 2010), and anticonvulsant effects (Lara et al., 2001) in rodents. GUO treatment exerts neuroprotection on Alzheimer's and Parkinson's disease in vivo models, ameliorating behavior, cognitive and motor function (Su et al., 2009; Massari et al., 2017; Lanznaster, et al., 2016; Marques et al., 2019; da Silva et al., 2020). In vitro studies showed that GUO modulates glutamate uptake, decreases the production of reactive oxygen species (ROS), improves mitochondrial function and presents anti-inflammatory properties (DalCim et al., 2012; Marques, et al., 2019; Frizzo et al., 2003; Dal-Cim et al., 2019). Regarding trophic effects, GUO increases the number of neurons in culture (Decker et al., 2019) and recently it was shown that GUO promotes neural stem cell proliferation and neuronal differentiation in vitro. Additionally, GUO in vivo treatment increases the number of dividing cells and also increases neurogenesis in the hippocampal dentate gyrus (Piermartiri et al., 2020).

\section{Guanosine Interaction Sites}

The intracellular signaling pathways related to GUO effects were the first targets to be identified. It was already shown that GUO effect on cell proliferation is dependent on cyclic AMP (cAMP) level increase (Gysbers and Rathbone 1996; Su et al., 2009). Also, some protective effects are directly related to Phosphoinositide 3-kinase/Protein kinase B (PI3K/Akt) pathway (Dal-Cim et al., 2013; Dal-Cim et al., 2012; Molz et al., 2011; D’Alimonte et al., 2007; Giuliani et al., 2015) and the mitogenactivated protein kinase/extracellular signal-regulated kinase (MAPK/ERK) pathway (Di Iorio et al., 2004; Dal-Cim et al., 2011). Recently, the involvement of protein kinase C (PKC) was also identified, as GUO effect of increasing the glial glutamate transporter-1 (Glt-1) membrane expression after an

Abbreviations: A1R, adenosine A1 receptors; A2AR, adenosine A2A receptors; cAMP, cyclic AMP; CGS21680, 4-(2-[7Amino-2-(2-furyl)[1,2,4] triazolo[2,3-a][1,3,5]triazin-5-ylamino]ethyl) phenol; CNS, central nervous system; DPCPX, 1,3dipropyl-8-cyclopentylxanthine; GPCR, G-protein coupled receptor; GUO, Guanosine; OGD, oxygen/glucose deprivation; ROS, reactive oxygen species; R-PIA, R(-)-N6-(2-phenylisopropyl)adenosine; SUMO, Small Ubiquitin-like Modifier. 
oxygen/glucose deprivation (OGD) protocol in astrocytes is abolished by PKC or MAPK/ERK inhibition (Dal-Cim et al., 2019).

Since GUO effects evoke intracellular signaling pathways, the need for a membrane receptor target is claimed. The effects of GUO are not altered by nucleosides transporter blockers (Oleskovicz et al., 2008; Giuliani et al., 2015; Decker et al., 2019), indicating that its effects are mediated through interaction with some molecular target at the cellular membrane not yet identified. Some studies pointed to a putative selective GUO receptor in rat brain membranes through $\left[{ }^{3} \mathrm{H}\right] \mathrm{GUO}$ binding analysis (Traversa et al., 2002; Traversa et al., 2003). Additionally, a study from Volpini and collaborators suggested GUO interaction with a G-protein coupled receptor (GPCR) (Volpini et al., 2011). In this line of evidence, the pharmacological blockade of GUO neuroprotective effect against ischemia-like in vitro protocol with Pertussis toxin also indicates a GPCR interaction (Dal-Cim et al., 2013). While the extracellular effects of purinergic adenine-based nucleotides and nucleoside are better characterized and their respective P2 and P1 receptors are recognized (Palmer and Stiles 1995), guanine-based purines are still orphan molecules.

Apart from the purinergic system, it was shown that GUO effects may depend on a potassium channel interaction. GUO effects of increasing cellular viability in hippocampal slices subjected to OGD and in SH-SY5Y neuroblastoma cells subjected to mitochondrial damage are blocked by large (big) conductance calcium-activated potassium channels (BK) inhibitors (Oleskovicz et al., 2008; Dal-Cim et al., 2012; DalCim et al., 2013). A couple of studies also suggested the relation of GUO effects with GPR23 (Di Liberto et al., 2012) or CD40 receptors (D'Alimonte et al., 2007). Although there is a suggestion for GUO receptors (a selective one, or other putative receptors), a great number of results support that GUO effects are mediated by adenosine receptors (Lanznaster, et al., 2016).

\section{Guanosine Interaction With Adenosine Receptors}

Adenosine plays a pivotal role as a neuromodulator and presents neurotrophic effects acting through its $\mathrm{P} 1$ receptors, which are composed of four different GPCRs $\left(A_{1} R, A_{2 A} R, A_{2 B} R\right.$, and $\left.A_{3} R\right)$. $A_{1} R$ and $A_{3} R$ are typically coupled to $G i$ proteins and thus inhibit adenylyl cyclase activity, whereas $A_{2 A} R$ and $A_{2 B} R$ are coupled to Gs proteins and increase the production of cAMP (Zimmermann, 2011). P1 receptors are expressed in neurons, astrocytes, oligodendrocytes and microglia and their stimulation activates multiple functions, such as synaptic plasticity and presynaptic neuromodulation (Daré et al., 2007; Burnstock, Fredholm, and Verkhratsky 2011; Burnstock and Ulrich 2011). In addition, $A_{1} R$ and $A_{2 A} R$ are the main responsible for adenosine actions on the CNS, while $A_{1} R$ is well expressed in the whole brain, $A_{2 A} R$ is enriched in some particular areas such as the striatum, hippocampus, raphe nuclei and locus coeruleus (Palmer and Stiles 1995). And, to our knowledge, besides one study showing that a preferential $\mathrm{A}_{2 \mathrm{~B}} \mathrm{R}$ antagonist partially decreased the mitogenic activity of GUO in astrocytes (Ciccarelli et al., 2000), only $A_{1} R$ and $A_{2 A} R$ have been related to GUO effects.

Some results directly imply GUO effects with the $A_{1} R$. Most of GUO known effects are abolished by previous incubation or treatment with the selective $\mathrm{A}_{1} \mathrm{R}$ antagonist $\mathrm{DPCPX}$. In vitro protocols of brain ischemia in hippocampus slices and cortical astrocyte cultures demonstrated that DPCPX abolished the protective effects of GUO in ROS production, glutamate uptake, and cell viability (Dal-Cim et al., 2013; Dal-Cim et al., 2019). Similarly, DPCPX prevented GUO protective effect against mitochondrial oxidative stress in human neuroblastoma $\mathrm{SH}$ SY5Y cells (Dal-Cim et al., 2012). Also, it was shown that in primary culture of both neurons and astrocytes, GUO increases global Small Ubiquitin-like MOdifier (SUMO)2/3-ylation at neuroprotective concentrations, an effect abolished by DPCPX preincubation (Zanella et al., 2020). In vivo protocols also display the same pattern related to $A_{1} R$. In a reserpine-treated mice protocol, where animals develop a parkinsonian tremor and striatal damage, GUO reverses the motor impairment and decreases ROS level in the striatum, but GUO efficacy is lost when animals are pretreated with DPCPX (Massari et al., 2020). In a traumatic brain injury model in rats, it was seen that mitochondrial dysfunction in the cerebral cortex is reversed by GUO treatment. However, this effect is no longer seen if the animals are pretreated with DPCPX (Gerbatin et al., 2019). In the same way, DPCPX reversed the anxiolytic-like effect induced by GUO, as well as the GUO capacity of decreasing the synaptosomal $\mathrm{K}^{+}$-stimulated glutamate release (Almeida et al., 2017). It is important to mention that DPCPX is also considered to be an inverse agonist of $A_{1} R$ (Weyler et al., 2006). Additionally, reports are now revealing some molecules that display a biased agonism (a ligand-dependent differential intracellular signaling) on $A_{1} R$, an issue that still needs additional studies (Vecchio et al., 2018). Taken together, these data could suggest that GUO effects are mediated by $A_{1} R$ activation. However, in heterologous transfection of $A_{1} R$ in HEK293 cells, GUO does not induce calcium mobilization as observed with an $A_{1} R$ agonist (R-PIA) treatment (as a positive control). Moreover, GUO has no effect upon R-PIA-inducing calcium mobilization through $A_{1} R$ (Lanznaster, et al., 2019).

Data regarding GUO dependence on the $A_{2 A} R$ signaling are conflicting. While most data show that antagonism of $\mathrm{A}_{2 \mathrm{~A}} \mathrm{R}$ has no impact on GUO promoting effects (Almeida et al., 2016; Almeida et al., 2017; Gerbatin et al., 2019; Massari et al., 2020; Zanella et al., 2020) some reports are showing otherwise (DalCim et al., 2012; Decker et al., 2019). Surprisingly, the pharmacological use of the $\mathrm{A}_{2 \mathrm{~A}} \mathrm{R}$ agonist CGS21680 shows a clear counteraction of GUO-mediating effects. Like the pretreatment with an $\mathrm{A}_{1} \mathrm{R}$ antagonist, $\mathrm{A}_{2 \mathrm{~A}} \mathrm{R}$ agonist also abolishes the protective effects of GUO over ROS production, glutamate uptake, and cell viability on those in vitro protocols of brain ischemia (Dal-Cim et al., 2013; Dal-Cim et al., 2019). Recently, it was seen that in mice that do not express $A_{2 A} R$ (i.e. $A_{2 A} \mathrm{R}$ Knock-out mice, $\mathrm{A}_{2} \mathrm{AR}^{-/-}$) the preventive GUO effect on ROS production and on cell viability is impaired (Lanznaster et al., 2019). Important to notice, GUO per se does not induce cAMP increase in HEK293 cells transfected with $A_{2 A} R$, neither 
interfere with cAMP level increase induced by the $\mathrm{A}_{2 \mathrm{~A}} \mathrm{R}$ agonist CGS 26180 (Lanznaster et al., 2019).

The dubious effect of GUO on adenosine receptors can also be interpreted through the oligomeric interaction of these receptors.

\section{Adenosine Receptors Forming-Oligomers}

The understanding of GPCRs physiology and pharmacology has changed in the last 2 decades. This is due to the growing evidence that they can form homomers (homo-oligomerization, from the same GPCRs) and heteromers (hetero-oligomerization of different GPCRs). This oligomerization induces changes in biochemical properties of GPCRs. It is well established that adenosine receptors can form oligomers among themselves and with receptors for other neurotransmitters, such as dopamine receptors (Ginés et al., 2000; Fuxe et al., 2005; Ciruela et al., 2006; Navarro, et al., 2018; Borroto-Escuela et al., 2018; Ferré and Ciruela 2019; Cortés et al., 2019). It is known that $A_{1}$ and $A_{2 A}$ receptors form functional oligomers with each other and that the $A_{1} R-A_{2 A} R$ heteromer plays an important role in modulating the control of cortico-striatal function (Ciruela et al., 2006). This control takes place through the activation of the presynaptic $A_{1} R$ or $A_{2 A} R$, which depends on the concentration of adenosine, as a low concentration would activate $A_{1} R$ while a high concentration would activate $A_{2 A} R$, resulting in a lesser or greater release of glutamate, respectively (Ciruela et al., 2006). Moreover, the $\mathrm{A}_{1} \mathrm{R}-\mathrm{A}_{2 \mathrm{~A}} \mathrm{R}$ heteromer seems to have a role in glutamate clearance by modulating the expression of the excitatory amino acid transporter 2 (EAAT2) in astrocytes (Hou et al., 2020). Also, adenosine interaction with $\mathrm{A}_{1} \mathrm{R}-\mathrm{A}_{2 \mathrm{~A}} \mathrm{R}$ heteromer in astrocytes has been shown to control extracellular gamma-aminobutyric acid (GABA) uptake via modulation of GABA transporters (Cristóvão-Ferreira et al., 2013). In this way, it is proposed that $A_{1} R-A_{2 A} R$ heteromer works as an adenosine concentration-sensing device that implies a cross-communication between $\mathrm{Gi}$ and $\mathrm{Gs}$ proteins guided by the $\mathrm{C}$-terminal tail of the $\mathrm{A}_{2 \mathrm{~A}} \mathrm{R}$ (Navarro, Cordomí, Brugarolas, et al., 2018).

The structure of $\mathrm{A}_{1} \mathrm{R}$ and $\mathrm{A}_{2 \mathrm{~A}} \mathrm{R}$ heterodimerization was recently proposed through in silico molecular modeling (Navarro et al., 2016; Navarro, Cordomí, Brugarolas, et al., 2018). $A_{1} R-A_{2 A} R$ heteromer may be organized as a tetramer structure composed of two homodimers of $\mathrm{A}_{1} \mathrm{R}$ and two homodimers of $\mathrm{A}_{2 \mathrm{~A}} \mathrm{R}$. The homodimerization of $\mathrm{A}_{1} \mathrm{R}$ and $\mathrm{A}_{2 \mathrm{~A}} \mathrm{R}$ occurs through the transmembrane (TM) 4/5 interface while the heterodimerization takes place through the TM 5/6 interface of these receptors (Navarro, Cordomí, Brugarolas, et al., 2018).

In this line, using an in vitro approach with transfected HEK293 cells, we recently showed that GUO-induced effects require both $A_{1} R$ and $A_{2 A} R$ co-expression. GUO was able to decrease $A_{2 A} R$ binding affinity and cAMP response evoked by a selective $A_{2 A} R$ ligand but only in cells expressing both $A_{1} R$ and $A_{2 A} R$. Also, GUO had no effect on $A_{1} R$ signaling in the presence or absence of $\mathrm{A}_{2 \mathrm{~A}} \mathrm{R}$ co-expression (Lanznaster et al., 2019). Thereby, we interpret that GUO interacts with the adenosine receptors as a heteromeric entity, thus the most adjusted hypothesis is that GUO could be acting as a negative

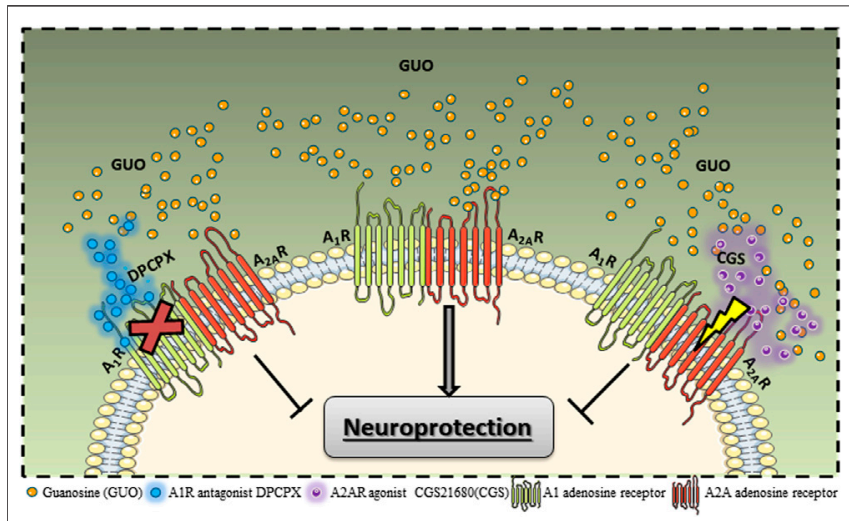

FIGURE 1 | Guanosine (GUO) interaction with adenosine $A_{1} R-A_{2 A} R$ heteromer. Studies with heterologous adenosine receptors transfection showed GUO effects on ligand binding to receptors and intracellular signaling activation require both $A_{1} R$ and $A_{2 A} R$ co-expression. Studies evaluating the neuroprotection promoted by GUO showed the protective is effect is abolished by $A_{2 A} R$ agonist (CGS21680) and $A_{1} R$ antagonist (DPCPX). This pharmacological modulation also points to an interaction with the $A_{1} R-A_{2 A} R$ heteromer.

modulator of $A_{2 A} R$, but only in the presence of $A_{1} R$. It is feasible to speculate that the physical interaction between $A_{1} R$ and $A_{2 A} R$ could lead to an increase of $A_{2 A} R$ affinity for GUO. CGS21680 could be directly interfering in the GUO signaling on $\mathrm{A}_{2 \mathrm{~A}} \mathrm{R}$, whereas $\mathrm{DPCPX}$ interacting with $\mathrm{A}_{1} \mathrm{R}$ may be responsible for allosteric modulation of GUO $A_{2 A} R$ affinity upon the $A_{1} R-A_{2 A} R$ heteromers (Figure 1). Indeed, GUO modulation over other adenosine-forming heteromers could not be discarded and might be different among brain structures, once that it may depend on differential receptors expression. Intriguingly, the GUO protective effect is lost in hippocampal but not in striatal slices from $\mathrm{A}_{2} \mathrm{AR}^{-/-}$mice (Lanznaster et al., 2019; Massari et al., 2020). Therefore, more studies regarding GUO interactions with adenosine oligomers are necessary.

\section{CONCLUSIONS AND PERSPECTIVES}

The increasing evidence supporting GUO protective action and trophic effects in the CNS are undeniable. This nucleoside is still considered an orphan neuromodulator, although its importance as an integrative molecule between purinergic and glutamatergic transmission. Some evidences suggest a selective GUO interaction site, whereas several studies show a dependence of GUO effects on adenosine receptors interaction. Considering the new paradigms related to adenosine receptors pharmacology (as allosterism, bias agonism and oligomeric interactions), there is a multitude of new interaction sites to be explored. These new insights of GUO interaction within GPCR heteromerization and the understanding of GUO effects on adenosine $A_{1} R-A_{2 A} R$ heteromers could open a new window in therapeutic approaches toward purinergic signaling. 


\section{AUTHOR CONTRIBUTIONS}

CT conceived the study. CM wrote the manuscript and composed the figure. CM, CT, MZ, and PD contributed in writing, editing and approved the submitted version of the manuscript.

\section{REFERENCES}

Almeida, R. F., Comasseto, D. D., Ramos, D. B., Hansel, G., Zimmer, E. R., Loureiro, S. O., et al. (2016). Guanosine anxiolytic-like effect involves adenosinergic and glutamatergic neurotransmitter systems. Mol. Neurobiol. 54, 423. doi:10.1007/s12035-015-9660-x

Almeida, R. F., Comasseto, D. D., Ramos, D. B., Hansel, G., Zimmer, E. R., Loureiro, S. O., et al. (2017). Guanosine anxiolytic-like effect involves adenosinergic and glutamatergic neurotransmitter systems. Mol. Neurobiol. 54 (1), 423-436. doi:10.1007/s12035-015-9660-x

Bettio, L. E. B., Cunha, M. P., Budni, J., Pazini, F. L., Oliveira, Á., Colla, A. R., et al. (2012). Guanosine produces an antidepressant-like effect through the modulation of NMDA receptors, nitric oxide-cGMP and PI3K/mTOR pathways. Behav. Brain Res. 234 (2), 137-148. doi:10.1016/j.bbr.2012. 06.021

Bettio, L. E. B., Freitas, A. E., Neis, V. B., Santos, D. B., Ribeiro, C. M., Rosa, P. B., et al. (2014). Guanosine prevents behavioral alterations in the forced swimming test and hippocampal oxidative damage induced by acute restraint stress. Pharmacol. Biochem. Behav. 127, 7-14. doi:10.1016/j.pbb.2014.10.002

Borroto-Escuela, D. O., Rodriguez, D., Romero-Fernandez, W., Kapla, J., Jaiteh, M., Ranganathan, A., et al. (2018). Mapping the interface of a GPCR dimer: a structural model of the A2A adenosine and D2 dopamine receptor heteromer. Front. Pharmacol. 9, 829. doi:10.3389/fphar.2018.00829

Burnstock, G., B. Fredholm, B., and Verkhratsky, A. (2011). Adenosine and ATP receptors in the brain. Ctmc 11 (8), 973-1011. doi:10.2174/ 156802611795347627

Burnstock, G., and Ulrich, H. (2011). Purinergic signaling in embryonic and stem cell development. Cell. Mol. Life Sci. 68 (8), 1369-1394. doi:10.1007/s00018010-0614-1

Ciccarelli, R., Di Iorio, P., D’Alimonte, I., Giuliani, P., Florio, T., Caciagli, F., et al. (2000). Cultured astrocyte proliferation induced by extracellular guanosine involves endogenous adenosine and is raised by the co-presence of microglia. Glia 29 (3), 202-211. doi:10.1002/(sici)1098-1136(20000201)29:3<202:aidglia2>3.0.co;2-c

Ciruela, F., Casadó, V., Rodrigues, R. J., Luján, R., Burgueño, J., Canals, M., et al. (2006). Presynaptic control of striatal glutamatergic neurotransmission by adenosine A1-A2A receptor heteromers. J. Neurosci. 26 (7), 2080-2087. doi:10.1523/JNEUROSCI.3574-05.2006

Cortés, A., Casadó-Anguera, V., Moreno, E., and Casadó, V. (2019). The heterotetrameric structure of the adenosine A1-dopamine D1 receptor complex: pharmacological implication for restless legs syndrome. $A d v$. Pharmacol. 84, 37-78. doi:10.1016/bs.apha.2019.01.001

Cristóvão-Ferreira, S., Navarro, G., Brugarolas, M., Pérez-Capote, K., Vaz, S. H., Fattorini, G., et al. (2013). A1R-A2AR heteromers coupled to Gs and Gi/0 proteins modulate GABA transport into astrocytes. Purinergic Signal. 9 (3), 433-449. doi:10.1007/s11302-013-9364-5

D’Alimonte, I., Flati, V., D’Auro, M., Toniato, E., Martinotti, S., Rathbone, M. P., et al. (2007). Guanosine inhibits CD40 receptor expression and function induced by cytokines and beta amyloid in mouse microglia cells. J. Immunol. 178 (2), 720-731. doi:10.4049/jimmunol.178.2.720

da Silva, J. S., Nonose, Y., Rohden, F., Lukasewicz Ferreira, P. C., Fontella, F. U., Rocha, A., et al. (2020). Guanosine neuroprotection of presynaptic mitochondrial calcium homeostasis in a mouse study with amyloid- $\beta$ oligomers. Mol. Neurobiol. 57 (11), 4790-4809. doi:10.1007/s12035-02002064-4

Dal-Cim, T., Ludka, F. K., Martins, W. C., Reginato, C., Parada, E., Egea, J., et al. (2013). Guanosine controls inflammatory pathways to afford neuroprotection of hippocampal slices under oxygen and glucose deprivation conditions. J. Neurochem. 126 (4), 437-450. doi:10.1111/jnc.12324

\section{FUNDING}

This article was partially supported by grants to PDI from the Italian Ministry of Education University and Research (MIUR).

Dal-Cim, T., Martins, W. C., Santos, A. R. S., and Tasca, C. I. (2011). Guanosine is neuroprotective against oxygen/glucose deprivation in hippocampal slices via large conductance $\mathrm{Ca} 2+$-activated $\mathrm{K}+$ channels, phosphatidilinositol-3 kinase/ protein kinase B pathway activation and glutamate uptake. Neuroscience 183, 212-220. doi:10.1016/j.neuroscience.2011.03.022

Dal-Cim, T., Molz, S., Egea, J., Parada, E., Romero, A., Budni, J., et al. (2012). Guanosine protects human neuroblastoma SH-SY5Y cells against mitochondrial oxidative stress by inducing heme oxigenase-1 via PI3K/Akt/ GSK-3 $\beta$ pathway. Neurochem. Int. 61 (3), 397-404. doi:10.1016/j.neuint.2012. 05.021

Dal-Cim, T., Poluceno, G. G., Lanznaster, D., de Oliveira, K. A., Nedel, C. B., and Tasca, C. I. (2019). Guanosine prevents oxidative damage and glutamate uptake impairment induced by oxygen/glucose deprivation in cortical astrocyte cultures: involvement of $\mathrm{A} 1$ and $\mathrm{A} 2 \mathrm{~A}$ adenosine receptors and PI3K, MEK, and PKC pathways. Purinergic Signal. 15, 465. doi:10.1007/s11302-01909679-w

Daré, E., Schulte, G., Karovic, O., Hammarberg, C., and Fredholm, B. B. (2007). Modulation of glial cell functions by adenosine receptors. Physiol. Behav. 92 (12), 15-20. doi:10.1016/j.physbeh.2007.05.031

Decker, H., Piermartiri, T. C. B., Nedel, C. B., Romão, L. F., Francisco, S. S., DalCim, T., et al. (2019). Guanosine and GMP increase the number of granular cerebellar neurons in culture: dependence on adenosine A2A and ionotropic glutamate receptors. Purinergic Signal. 15 (4), 439-450. doi:10.1007/s11302019-09677-y

Di Iorio, P., Ballerini, P., Traversa, U., Nicoletti, F., D’Alimonte, I., Kleywegt, S., et al. (2004). The antiapoptotic effect of guanosine is mediated by the activation of the PI 3-kinase/AKT/PKB pathway in cultured rat astrocytes. Glia 46 (4), 356-368. doi:10.1002/glia.20002

Di Liberto, V., Garozzo, R., Grillo, M., Mudo, G., Caciagli, F., Condorelli, D. F., et al. (2012). Identification of GPR23/LPA4 as a candidate G protein-coupled receptor for guanosine. Acta Physiol. 206, 692.

Ferré, S., and Ciruela, F. (2019). Functional and neuroprotective role of striatal adenosine A2AReceptor heterotetramers. J. Caffeine Adenosine Res. 9 (3), 89-97. doi:10.1089/caff.2019.0008

Frizzo, M. E., Antunes Soares, F. A., Dall'Onder, L. P., Lara, D. R., Swanson, R. A., and Souza, D. O. (2003). Extracellular conversion of guanine-based purines to guanosine specifically enhances astrocyte glutamate uptake. Brain Res. 972 (12), 84-89. doi:10.1016/s0006-8993(03)02506-x

Fuxe, K., Ferré, S., Canals, M., Torvinen, M., Terasmaa, A., Marcellino, D., et al. (2005). Adenosine A2A and dopamine D2 heteromeric receptor complexes and their function. Jmn 26 (2-3), 209-220. doi:10.1385/JMN:26:2-3:209

Gerbatin, R. R., Dobrachinski, F., Cassol, G., Soares, F. A. A., and Royes, L. F. F. (2019). A1 rather than A2A adenosine receptor as a possible target of Guanosine effects on mitochondrial dysfunction following Traumatic Brain Injury in rats. Neurosci. Lett. 704, 141-144. doi:10.1016/j.neulet.2019.04.014

Ginés, S., Hillion, J., Torvinen, M., Le Crom, S., Casadó, V., Canela, E. I., et al. (2000). Dopamine D1 and adenosine A1 receptors form functionally interacting heteromeric complexes. Proc. Natl. Acad. Sci. 97 (15), 8606-8611. doi:10.1073/pnas.150241097

Giuliani, P., Ballerini, P., Buccella, S., Ciccarelli, R., Rathbone, M. P., Romano, S et al. (2014). Guanosine protects glial cells against 6-hydroxydopamine toxicity. Adv. Exp. Med. Biol. 837, 23-33. doi:10.1007/5584_2014_73

Gysbers, J. W., and Rathbone, M. P. (1996). Neurite outgrowth in PC12 cells is enhanced by guanosine through both cAMP-dependent and -independent mechanisms. Neurosci. Lett. 220 (3), 175-178. doi:10.1016/s0304-3940(96) 13253-5

Hepler, J. R., and Gilman, A. G. (1992). G proteins. Trends Biochem. Sci. 17 (10), 383-387. doi:10.1016/0968-0004(92)90005-t

Hou, X., Li, Y., Huang, Y., Zhao, H., and Gui, L. (2020). Adenosine receptor A1-a2a heteromers regulate EAAT2 expression and glutamate uptake via YY1-induced 
repression of PPAR $\gamma$ transcription. PPAR Res. 2020, 1. doi:10.1155/2020/ 2410264

Lanznaster, D., Dal-Cim, T., Piermartiri, T. C. B., and Tasca, C. I. (2016). Guanosine: a neuromodulator with therapeutic potential in brain disorders. A\&D 7 (5), 657-679. doi:10.14336/AD.2016.0208

Lanznaster, D., Mack, J. M., Coelho, V., Ganzella, M., Almeida, R. F., Dal-Cim, T., et al. (2016). Guanosine prevents anhedonic-like behavior and impairment in hippocampal glutamate transport following amyloid- $\beta 1-40$ administration in mice. Mol. Neurobiol. 54, 5482. doi:10.1007/s12035-016-0082-1

Lanznaster, D., Massari, C. M., Marková, V., Šimková, T., Duroux, R., Jacobson, K. A., et al. (2019). Adenosine A1-a2a receptor-receptor interaction: contribution to guanosine-mediated effects. Cells 8 (12), 1630. doi:10.3390/cells8121630

Lara, D. R., Schmidt, A. P., Frizzo, M. E. S., Burgos, J. S., Ramírez, G., and Souza, D. O. (2001). Effect of orally administered guanosine on seizures and death induced by glutamatergic agents. Brain Res. 912 (2), 176-180. doi:10.1016/ s0006-8993(01)02734-2

Marques, N. F., Binder, L. B., Roversi, K., Sampaio, T. B., Constantino, L. C., Prediger, R. D., et al. (2019). Guanosine prevents depressive-like behaviors in rats following bilateral dorsolateral striatum lesion induced by 6-hydroxydopamine. Behav. Brain Res. 372, 112014. doi:10.1016/j.bbr.2019.112014

Marques, N. F., Massari, C. M., and Tasca, C. I. (2019). Guanosine protects striatal slices against 6-OHDA-induced oxidative damage, mitochondrial dysfunction, and ATP depletion. Neurotox Res. 35 (2), 475-483. doi:10.1007/s12640-0189976-1

Massari, C. M., Constantino, L. C., Marques, N. F., Binder, L. B., Valle-León, M., López-Cano, M., et al. (2020). Involvement of adenosine A1 and A2A receptors on guanosine-mediated anti-tremor effects in reserpinized mice. Purinergic Signal. 16, 379. doi:10.1007/s11302-020-09716-Z

Massari, C. M., López-Cano, M., Núñez, F., Fernández-Dueñas, V., Tasca, C. I., and Ciruela, F. (2017). Antiparkinsonian efficacy of guanosine in rodent models of movement disorder. Front. Pharmacol. 8, 700. doi:10.3389/fphar.2017.00700

Molz, S., Dal-Cim, T., Budni, J., Martín-de-Saavedra, M. D., Egea, J., Romero, A., et al. (2011). Neuroprotective effect of guanosine against glutamate-induced cell death in rat hippocampal slices is mediated by the phosphatidylinositol-3 kinase/Akt/ glycogen synthase kinase $3 \beta$ pathway activation and inducible nitric oxide synthase inhibition. J. Neurosci. Res. 89 (9), 1400-1408. doi:10.1002/jnr.22681

Navarro, G., Cordomí, A., Brugarolas, M., Moreno, E., Aguinaga, D., Pérez-Benito, L., et al. (2018). Cross-communication between Gi and Gs in a G-proteincoupled receptor heterotetramer guided by a receptor $\mathrm{C}$-terminal domain. $B M C$ Biol. 16 (1), 24. doi:10.1186/s12915-018-0491-x

Navarro, G., Cordomí, A., Casadó-Anguera, V., Moreno, E., Cai, N.-S., Cortés, A., et al. (2018). Evidence for functional pre-coupled complexes of receptor heteromers and adenylyl cyclase. Nat. Commun. 9 (1), 1242. doi:10.1038/ s41467-018-03522-3

Navarro, G., Cordomí, A., Zelman-Femiak, M., Brugarolas, M., Moreno, E., Aguinaga, D., et al. (2016). Quaternary structure of a G-protein-coupled receptor heterotetramer in complex with Gi and Gs. BMC Biol. 14, 26. doi:10.1186/s12915-016-0247-4

Oleskovicz, S. P. B., Martins, W. C., Leal, R. B., and Tasca, C. I. (2008). Mechanism of guanosine-induced neuroprotection in rat hippocampal slices submitted to oxygen-glucose deprivation. Neurochem. Int. 52 (3), 411-418. doi:10.1016/j. neuint.2007.07.017
Palmer, T. M., and Stiles, G. L. (1995). Adenosine receptors. Neuropharmacology 34 (7), 683-694. doi:10.1016/0028-3908(95)00044-7

Piermartiri, T. C. B., Dos Santos, B., Barros-Aragão, F. G. Q., Prediger, R. D., and Tasca, C. I. (2020). Guanosine promotes proliferation in neural stem cells from Hippocampus and neurogenesis in adult mice. Mol. Neurobiol. 57 (9), 3814-3826. doi:10.1007/s12035-020-01977-4

Schmidt, A., Böhmer, A., Schallenberger, C., Antunes, C., Tavares, R., Wofchuk, S., et al. (2010). Mechanisms involved in the antinociception induced by systemic administration of guanosine in mice. Br. J. Pharmacol. 159 (6), 1247-1263. doi:10.1111/j.1476-5381.2009.00597.x

Schmidt, A. P., Lara, D. R., and Souza, D. O. (2007). Proposal of a guanine-based purinergic system in the mammalian central nervous system. Pharmacol. Ther. 116 (3), 401-416. doi:10.1016/j.pharmthera.2007.07.004

Su, C., Elfeki, N., Ballerini, P., D’Alimonte, I., Bau, C., Ciccarelli, R., et al. (2009). Guanosine improves motor behavior, reduces apoptosis, and stimulates neurogenesis in rats with parkinsonism. J. Neurosci. Res. 87 (3), 617-625. doi:10.1002/jnr.21883

Traversa, U., Bombi, G., Camaioni, E., Macchiarulo, A., Costantino, G., Palmieri, C., et al. (2003). Rat brain guanosine binding site. Bioorg. Med. Chem. 11 (24), 5417-5425. doi:10.1016/j.bmc.2003.09.043

Traversa, U., Bombi, G., Iorio, P. D., Ciccarelli, R., Werstiuk, E. S., and Rathbone, M. P. (2002). Specific [3 H]-guanosine binding sites in rat brain membranes. $\mathrm{Br}$. J. Pharmacol. 135 (4), 969-976. doi:10.1038/sj.bjp.0704542

Vecchio, E. A., Baltos, J.-A., Nguyen, A. T. N., Christopoulos, A., White, P. J., and May, L. T. (2018). New paradigms in adenosine receptor pharmacology: allostery, oligomerization and biased agonism. Br. J. Pharmacol. 175 (21), 4036-4046. doi:10.1111/bph.14337

Volpini, R., Marucci, G., Buccioni, M., Dal Ben, D., Lambertucci, C., Lammi, C., et al. (2011). Evidence for the existence of a specific $\mathrm{G}$ protein-coupled receptor activated by guanosine. ChemMedChem 6 (6), 1074-1080. doi:10.1002/cmdc. 201100100

Weyler, S., Fülle, F., Diekmann, M., Schumacher, B., Hinz, S., Klotz, K.-N., et al. (2006). Improving potency, selectivity, and water solubility of adenosine A1 receptor antagonists: xanthines modified at position 3 and related pyrimido [1,2,3-cd]purinediones. ChemMedChem 1 (8), 891-902. doi:10.1002/cmdc. 200600066

Zanella, C. A., Tasca, C. I., Henley, J. M., Wilkinson, K. A., and Cimarosti, H. I. (2020). Guanosine modulates SUMO2/3-ylation in neurons and astrocytes via adenosine receptors. Purinergic Signal. 16 (3), 439-450. doi:10.1007/s11302020-09723-0

Conflict of Interest: The authors declare that the research was conducted in the absence of any commercial or financial relationships that could be construed as a potential conflict of interest.

Copyright (c) 2021 Massari, Zuccarini, Di Iorio and Tasca. This is an open-access article distributed under the terms of the Creative Commons Attribution License (CC $B Y$ ). The use, distribution or reproduction in other forums is permitted, provided the original author(s) and the copyright owner(s) are credited and that the original publication in this journal is cited, in accordance with accepted academic practice. No use, distribution or reproduction is permitted which does not comply with these terms. 